\title{
WEAK SOLUTIONS OF GEOMETRIC FLOWS ASSOCIATED TO INTEGRO-DIFFERENTIAL HARMONIC MAPS
}

\author{
ARMIN SCHIKORRA, YANNICK SIRE, AND CHANGYOU WANG
}

ABstract. The purpose of this note is to prove the existence of global weak solutions to the flow associated to integro-differential harmonic maps into spheres and Riemannian homogeneous manifolds.

\section{Contents}

1. Introduction

2. Preliminaries

3. Proof of Theorem 1.1; Step 1

4. Proof of Theorem 1.1. Step 2 - the case of a sphere

5. Proof of Theorem 1.1. Step 2 - the case of homogeneous Riemannian manifolds

References

\section{INTRODUCTION}

Let $\mathcal{N}$ be a compact smooth connected manifold without boundary, isometrically embedded into $\mathbb{R}^{L}$, and $\Omega$ be a smooth bounded domain of $\mathbb{R}^{n}$ with $n \geq 1$. Consider the Dirichlet energy functional

$$
E_{2}(u)=\int_{\Omega}|\nabla u|^{2} d x,
$$

where $u$ maps $\Omega$ into $\mathcal{N}$. The harmonic map flow is then the flow defined by: given $u_{0}$ an initial data in a suitable functional space, consider

$$
\begin{cases}\partial_{t} u+E_{2}^{\prime}(u, \cdot) \perp T_{u} \mathcal{N} & \text { in } \Omega \times(0, \infty), \\ u(\cdot, 0)=u_{0} & \text { in } \Omega, \\ u(\cdot, t)=u_{1} & \text { on } \partial \Omega \times(0, \infty) .\end{cases}
$$


In the previous expression, $E_{2}^{\prime}$ denotes the Frechet derivative of the functional $E_{2}$. This flow has been studied in many papers (see e.g. [Che89, CS89, Str88] and the monograph [LW08]).

In the present paper, we investigate the flow associated to the critical points of the following type of nonlocal energy:

$$
E_{s, p}(u)=\frac{1}{p} \int_{\Omega \times \Omega} \frac{|u(x)-u(y)|^{p}}{|x-y|^{n+s p}} d x d y\left(\equiv[u]_{\dot{W}^{s, p}(\Omega)}^{p}\right),
$$

where $1<p<+\infty, s \in(0,1), \Omega$ is a Lipschitz domain in $\mathbb{R}^{n}$ (possibly the whole $\mathbb{R}^{n}$ ), $n \geq 1$, and $u$ takes values into $\mathcal{N} \subset \mathbb{R}^{L}$. We define the Sobolev space

$$
W^{s, p}(\Omega, \mathcal{N})=\left\{u \in W^{s, p}\left(\Omega, \mathbb{R}^{L}\right): u(x) \in \mathcal{N} \text { for a.e. } x \in \Omega\right\} .
$$

The quantity $E_{s, p}(u)$ is known as the Gagliardo norm of $u$ and has been investigated in the context of harmonic maps (i.e. with the sidecondition that $u$ maps into $\mathcal{N}$ ) by several authors. In the case, $n=1$, $s=\frac{1}{2}$ and $p=2$, the regularity of such maps has been obtained in [DLR11b, DLR11a]. In the case of general $n \geq 2, s=\frac{n}{2}$ it has been investigated in [Sch12, DL13]. In the case, $p=\frac{n}{s}$ for any $n \geq 1$ and $s \in(0,1)$, this has been investigated by one of the authors [Sch15] in the case of $\mathcal{N}=\mathbb{S}^{L-1}$. In all the previous results, the exponent $p$ is the conformal exponent $p=\frac{n}{s}$, and in this case one expects regularity to hold everywhere. If $p<\frac{n}{s}$ one expects only partial regularity and no results are available in the literature in this case. In the present paper, we introduce and investigate the flow associated to these maps in all dimensions and for any $p$ and $s$ in the above ranges.

Remark 1.1. In the paper [MS15], a Ginzburg-Landau approximation of $\frac{1}{2}$-harmonic maps is performed. Furthermore, it appears that $\frac{1}{2}$ harmonic maps can be formulated as harmonic maps with free boundaries (see [HL89]). This approach is a new feature of fractional harmonic maps, that we will not exploit here.

We now recall the flow associated with $E_{s, p}(\cdot)$ for maps from $\Omega$ to $\mathcal{N}:$ given $u_{0}: \Omega \rightarrow \mathcal{N}$ an initial data in a suitable functional space, consider $u: \Omega \times[0, \infty) \rightarrow \mathcal{N}$ satisfying

$$
\begin{cases}\partial_{t} u+E_{s, p}^{\prime}(u, \cdot) \perp T_{u} \mathcal{N} & \text { in } \Omega \times(0, \infty), \\ u(\cdot, 0)=u_{0} & \text { in } \Omega, \\ u(\cdot, t)=u_{1} & \text { on } \partial \Omega \times(0, \infty) .\end{cases}
$$


Here, $E_{s, p}^{\prime}$ denotes the Frechet derivative of $E_{s, p}$ in the function space $W^{s, p}\left(\Omega, \mathbb{R}^{L}\right)$ : for $v \in W^{s, p}(\Omega, \mathcal{N})$ and $\varphi \in W_{0}^{s, p}\left(\Omega, \mathbb{R}^{L}\right)$,

$$
E_{s, p}^{\prime}(v, \varphi)=\int_{\Omega^{2}} \frac{|v(x)-v(y)|^{p-2}(v(x)-v(y))(\varphi(x)-\varphi(y))}{|x-y|^{n+s p}} .
$$

Our main result is the following. It provides the existence of global weak solutions to the flow (3) .

Theorem 1.1. Let $\mathcal{N}=\mathbb{S}^{L-1} \subset \mathbb{R}^{L}$ be the unit sphere or $\mathcal{N}$ be a Riemannian homogeneous manifold that is equivariantly embedded into $\mathbb{R}^{L}$. For any $u_{0} \in W^{s, p}(\Omega, \mathcal{N})$, there exists $u: \Omega \times(0, \infty) \rightarrow \mathcal{N}$ such that

$$
\begin{cases}\partial_{t} u+E_{s, p}^{\prime}(u, \cdot) \perp T_{u} \mathcal{N} & \text { in } \Omega \times(0, \infty), \\ u(\cdot, 0)=u_{0} & \text { in } \Omega,\end{cases}
$$

in the following sense:

- $u \in L^{\infty}\left(0, \infty ; W^{s, p}(\Omega)\right)$.

- $\partial_{t} u \in L^{2}\left(0, \infty ; L^{2}(\Omega)\right)$.

- $u$ satisfies the first equation in (5) in $\mathcal{D}^{\prime}(\Omega \times(0, \infty))$, and

$$
\lim _{t \rightarrow 0^{+}}\left\|u(\cdot, t)-u_{0}\right\|_{L^{2}(\Omega)}=0 .
$$

The attentive reader might have noticed that in (5) the third equation of (3) is missing. In our construction one can easily prescribe the boundary $u_{1}$ on $\partial \Omega$ in a distributional sense. However, if $s<1 / p$ this is meaningless, since the trace of $W^{s, p}(\Omega)$-functions belongs to $W^{s-1 / p, p}(\partial \Omega)$. One way to avoid this issue is to replace the third equation in (3) by

$$
u(\cdot, t)=u_{1} \quad \text { on }\left(\mathbb{R}^{n} \backslash \Omega\right) \times(0, \infty),
$$

and then find a flow $u:(0,+\infty) \rightarrow W^{s, p}\left(\mathbb{R}^{n}, \mathcal{N}\right)$. This is an easy adaptation of the arguments we present below, and we leave it to the interested reader.

Let us remark that in PG1] they studied the fractional LandauLifshitz-Gilbert equation and in particular obtain Theorem 1.1 in the case of the 2-sphere. Also, we would like to point out that the existence of global weak solutions to the $p$-harmonic maps to $\mathbb{S}^{L-1}$ has been proven by [Che89] and [CHH94].

\footnotetext{
${ }^{1}$ See (21) in Section 5.
} 


\section{Preliminaries}

We consider $\Omega \subset \mathbb{R}^{n}$ to be bounded. A simple adaption of the argument provides the case $\Omega=\mathbb{R}^{n}$.

Lemma 2.1. Let $\Omega \subset \mathbb{R}^{n}$ be a bounded domain and $K \subset \mathbb{R}^{L}$ a compact set. For any $s>0, p \in(1, \infty)$, and any $q \in[1, \infty), W^{s, p}(\Omega, K)$ is embedded compactly into $L^{q}(\Omega, K)$.

Proof. By interpolation we know that $W^{s, p}\left(\Omega, \mathbb{R}^{L}\right)$ is embedded compactly into $L^{1}\left(\Omega, \mathbb{R}^{L}\right)$. On the other hand, $W^{s, p}(\Omega, K) \subset L^{\infty}(\Omega)$ by the boundedness of $K$. In particular, $W^{s, p}(\Omega, K)$ is embedded compactly into $L^{q}(\Omega, K)$ for all $q \in[1, \infty)$.

For the classical $p$-Laplacian, it is not true that $f_{k} \rightarrow f$ weakly in $W^{1, p}(\Omega)$ implies that $\left|\nabla f_{k}\right|^{p-2} \nabla f_{k} \rightarrow|\nabla f|^{p-2} \nabla f$ weakly in $L^{\frac{p}{p-1}}(\Omega)$, i.e.,

$$
\lim _{k \rightarrow \infty} \int_{\Omega}\left|\nabla f_{k}\right|^{p-2} \nabla f_{k} \nabla \varphi=\int_{\Omega}|\nabla f|^{p-2} \nabla f \nabla \varphi, \varphi \in W^{1, p}(\Omega) .
$$

A nice feature of the fractional p-Laplacian is that an analogue is actually true. In fact, denote

$$
\Omega^{2}=\Omega \times \Omega, \Omega_{T}=\Omega \times[0, T], \text { and } \Omega_{T}^{2}=\Omega^{2} \times[0, T] .
$$

For $0<T \leq \infty$, we have the following property.

Lemma 2.2. For any $0<T<\infty$, assume that $f_{k} \rightarrow f$ a.e. in $\Omega_{T}$, $f_{k} \rightarrow f$ in $L^{p}\left(0, T ; W^{s, p}(\Omega)\right)$. Also assume that $\left\|g_{k}\right\|_{L^{\infty}\left(\Omega_{T}\right)}$ is bounded and $g_{k} \rightarrow g$ a.e. in $\Omega_{T}$. Then for any $\varphi \in L^{p}\left(0, T ; W^{s, p}(\Omega)\right)$ it holds that

$$
\begin{aligned}
& \int_{\Omega_{T}^{2}} \frac{\left|f_{k}(x, t)-f_{k}(y, t)\right|^{p-2}\left(f_{k}(x, t)-f_{k}(y, t)\right) g_{k}(y, t)(\varphi(x, t)-\varphi(y, t))}{|x-y|^{n+s p}} \\
& \stackrel{k \rightarrow \infty}{\longrightarrow} \\
& \int_{\Omega_{T}^{2}} \frac{|f(x, t)-f(y, t)|^{p-2}(f(x, t)-f(y, t)) g(y, t)(\varphi(x, t)-\varphi(y, t))}{|x-y|^{n+s p}} .
\end{aligned}
$$

Proof. From the assumptions on $f_{k}$ and $g_{k}$, we have that

$$
\begin{gathered}
c_{k}(x, y, t):=\frac{\left|f_{k}(x, t)-f_{k}(y, t)\right|^{p-2}\left(f_{k}(x, t)-f_{k}(y, t)\right) g_{k}(y, t)}{|x-y|^{\left(\frac{n}{p}+s\right)(p-1)}} \\
\stackrel{k \rightarrow \infty}{\longrightarrow} c(x, y, t):=\frac{|f(x, t)-f(y, t)|^{p-2}(f(x, t)-f(y, t)) g(y, t)}{|x-y|^{\left(\frac{n}{p}+s\right)(p-1)}},
\end{gathered}
$$


for a.e. $(x, y, t) \in \Omega_{T}^{2}$. Furthermore, direct calculations imply that

$$
\int_{\Omega_{T}^{2}}\left|c_{k}(x, y, t)\right|^{\frac{p}{p-1}} \leq C\left\|g_{k}\right\|_{L^{\infty}\left(\Omega_{T}\right)}^{\frac{p}{p-1}}\left\|f_{k}\right\|_{L^{p}\left(0, T ; W^{s, p}(\Omega)\right)}^{p} \leq C .
$$

We conclude that $c_{k}(x, y, t) \rightarrow c(x, y, t)$ in $L^{\frac{p}{p-1}}\left(\Omega_{T}^{2}\right)$ as $k \rightarrow \infty$. On other hand, if we set

$$
\Phi(x, y, t):=\frac{\varphi(x, t)-\varphi(y, t)}{|x-y|^{\frac{n}{p}+s}},(x, y, t) \in \Omega_{T}^{2},
$$

then $\Phi \in L^{p}\left(\Omega_{T}^{2}\right)$ and

$$
\|\Phi\|_{L^{p}\left(\Omega_{T}^{2}\right)} \leq C\|\varphi\|_{L^{p}\left(0, T ; W^{s, p}(\Omega)\right)}^{p} .
$$

Thus we obtain

$$
\int_{\Omega_{T}^{2}} c_{k}(x, y, t) \Phi(x, y, t) \stackrel{k \rightarrow \infty}{\longrightarrow} \int_{\Omega_{T}^{2}} c(x, y, t) \Phi(x, y, t) .
$$

This completes the proof.

As a direct consequence of Lemma 2.2, we have

Corollary 2.3. Let $f_{k} \rightarrow f$ in $W^{s, p}(\Omega)$, then for any $\varphi \in W^{s, p}(\Omega)$

$$
\begin{aligned}
& \lim _{k \rightarrow \infty} \int_{\Omega^{2}} \frac{\left|f_{k}(x)-f_{k}(y)\right|^{p-2}\left(f_{k}(x)-f_{k}(y)\right)(\varphi(x)-\varphi(y))}{|x-y|^{n+s p}} \\
& =\int_{\Omega^{2}} \frac{|f(x)-f(y)|^{p-2}(f(x)-f(y))(\varphi(x)-\varphi(y))}{|x-y|^{n+s p}} .
\end{aligned}
$$

We also need the following fact in the proof of Theorem 1.1.

Lemma 2.4. The functional $E_{s, p}$ is sequentially lower semi-continuous with respect to the weak topology on $W^{s, p}\left(\Omega, \mathbb{R}^{L}\right)$.

Proof. Assume that $f_{k} \rightarrow f$ in $W^{s, p}\left(\Omega, \mathbb{R}^{L}\right)$. Now we use a duality argument: It holds that

$$
\begin{aligned}
& \left(\int_{\Omega^{2}} \frac{|f(x)-f(y)|^{p}}{|x-y|^{n+s p}}\right)^{\frac{p}{p-1}} \\
& \leq \sup _{\varphi} \int_{\Omega^{2}} \frac{|f(x)-f(y)|^{p-2}(f(x)-f(y))(\varphi(x)-\varphi(y))}{|x-y|^{n+s p}},
\end{aligned}
$$

where the supremum is taken over $\varphi \in W^{s, p}\left(\Omega, \mathbb{R}^{L}\right)$, with $\|\varphi\|_{W^{s, p}(\Omega)} \leq$ 1. In particular, for any $\varepsilon>0$ we find some $\varphi_{\varepsilon} \in W^{s, p}\left(\Omega, \mathbb{R}^{L}\right)$, with 
$\left\|\varphi_{\varepsilon}\right\|_{W^{s, p}(\Omega)} \leq 1$, such that

$$
\begin{aligned}
& \left(\int_{\Omega^{2}} \frac{|f(x)-f(y)|^{p}}{|x-y|^{n+s p}} d x d y\right)^{\frac{p}{p-1}} \\
\leq & (1+\varepsilon) \int_{\Omega^{2}} \frac{|f(x)-f(y)|^{p-2}(f(x)-f(y))\left(\varphi_{\varepsilon}(x)-\varphi_{\varepsilon}(y)\right)}{|x-y|^{n+s p}} .
\end{aligned}
$$

By Lemma 2.2, the right-hand side of the above inequality is equal to

$$
(1+\varepsilon) \lim _{k \rightarrow \infty} \int_{\Omega^{2}} \frac{\left|f_{k}(x)-f_{k}(y)\right|^{p-2}\left(f_{k}(x)-f_{k}(y)\right)\left(\varphi_{\varepsilon}(x)-\varphi_{\varepsilon}(y)\right)}{|x-y|^{n+s p}},
$$

which, by Hölder's inequality, can be bounded by

$$
\leq(1+\varepsilon) \liminf _{k \rightarrow \infty}\left(\int_{\Omega^{2}} \frac{\left|f_{k}(x)-f_{k}(y)\right|^{p}}{|x-y|^{n+s p}} d x d y\right)^{\frac{p}{p-1}} .
$$

Sending $\varepsilon \rightarrow 0$, we then obtain the conclusion.

\section{Proof of Theorem 1.1: Step 1}

Step 1 is the time discretization of the flow (3). This step works for any compact Riemannian manifold $\mathcal{N} \subset \mathbb{R}^{L}$ without boundary. To the best of our knowledge this idea is due to an unpublished work by Kukuchi. Fix $h>0$. Starting from $u_{0} \in W^{s, p}(\Omega, \mathcal{N})$, for $k \geq 1$ we define $u^{k} \in W^{s, p}(\Omega, \mathcal{N})$ to be a minimizer of $\mathcal{E}_{k}$ in the space of maps $v \in W^{s, p}(\Omega, \mathcal{N})$, where

$$
\mathcal{E}_{k}(v):=E_{s, p}(v)+\int_{\Omega} \frac{\left|v-u^{k-1}\right|^{2}}{2 h} .
$$

This existence of $u^{k}$ follows from the direct method of calculus of variations, since $\mathcal{E}_{k}(\cdot)$ is coercive and sequentially lower semicontinuous with respect to the weak topology in $W^{s, p}(\Omega)$. Also observe the compactness of $\mathcal{N}$ guarantees that $u^{k}(x) \in \mathcal{N}$ for a.e. $x \in \Omega$, see Lemma 2.1.

By direct calculations, $u^{k}$ satisfies the Euler-Lagrange equation:

$$
\begin{aligned}
& \int_{\Omega} \frac{u^{k}-u^{k-1}}{h} \varphi^{k} \\
& =-\int_{\Omega^{2}} \frac{\left|u^{k}(x)-u^{k}(y)\right|^{p-2}\left(u^{k}(x)-u^{k}(y)\right)\left(\varphi^{k}(x)-\varphi^{k}(y)\right)}{|x-y|^{n+s p}}
\end{aligned}
$$


where $\varphi^{k}=P_{\mathcal{N}}\left(u^{k}\right)(\psi), P_{\mathcal{N}}(y): \mathbb{R}^{L} \rightarrow T_{y} \mathcal{N}$, for $y \in \mathcal{N}$, is the orthogonal projection 2 , and $\psi \in C_{0}^{\infty}\left(\Omega, \mathbb{R}^{L}\right)$.

It is clear, by the minimality and comparison, that for $j=1, \cdots, k$,

$$
\mathcal{E}_{j}\left(u^{j}\right)=E_{s, p}\left(u^{j}\right)+\int_{\Omega} \frac{\left|u^{j}-u^{j-1}\right|^{2}}{2 h} \leq \mathcal{E}_{j}\left(u^{j-1}\right)=E_{s, p}\left(u^{j-1}\right) .
$$

Taking summation over $j=1, \cdots, k$, we obtain that

$$
E_{s, p}\left(u^{k}\right)+\sum_{j=1}^{k} \int_{\Omega} \frac{\left|u^{j}-u^{j-1}\right|^{2}}{2 h} \leq E_{s, p}\left(u^{0}\right), \forall k \geq 1 .
$$

Here we denote $u^{0}=u_{0}$. Now we define $u^{h}: \Omega \times[0, \infty) \rightarrow \mathcal{N}$ and $v^{h}: \Omega \times[0, \infty) \rightarrow \mathbb{R}^{L}$ by letting

$$
\left\{\begin{array}{l}
u^{h}(x, t)=u^{k}(x) \\
v^{h}(x, t)=\frac{t-k h}{h} u^{k+1}(x)+\frac{(k+1) h-t}{h} u^{k}(x),
\end{array}\right.
$$

when $x \in \Omega, t \in[k h,(k+1) h)$ for some $k \geq 0$.

It follows from (7) that for any $h>0$, the following energy inequality holds:

$$
E_{s, p}\left(u^{h}(\cdot, t)\right)+\frac{1}{2} \int_{0}^{t} \int_{\Omega}\left|\partial_{t} v^{h}\right|^{2} \leq E_{s, p}\left(u_{0}\right), \forall t>0 .
$$

Since $\mathcal{N}$ is compact, it is easy to see that there exists $C>0$ depending only on $\mathcal{N}$ such that for any $h>0$,

$$
\left|v^{h}(x, t)\right| \leq C, \forall x \in \Omega, t>0 .
$$

Moreover, for any $h>0$ and $t>0$, we have, by convexity and (7),

$$
\begin{aligned}
E_{s, p}\left(v^{h}(\cdot, t)\right) & \leq \frac{t-k h}{h} E_{s, p}\left(u^{k+1}\right)+\frac{(k+1) h-t}{h} E_{s, p}\left(u^{k}\right) \\
& \leq E_{s, p}\left(u_{0}\right)
\end{aligned}
$$

where $k \geq 0$ is chosen so that $k h \leq t<(k+1) h$.

It follows from (8), (9), and (10) that there exist $u, v: \Omega \times(0, \infty) \rightarrow$ $\mathbb{R}^{L}$ such that after passing to a subsequence,

$v^{h} \rightarrow v$ in $L_{\text {loc }}^{2}(\Omega \times(0, \infty)),\left(\partial_{t} v^{h}, \nabla v^{h}\right) \rightarrow\left(\partial_{t} v, \nabla v\right)$ in $L_{\text {loc }}^{2}(\Omega \times(0, \infty))$, and

$$
u^{h} \rightarrow u \text { in } L_{\mathrm{loc}}^{p}\left((0, \infty), W^{s, p}(\Omega)\right),
$$

as $h \rightarrow 0$.

\footnotetext{
${ }^{2}$ It is well-known that there exists small $\delta>0$ depending only on $\mathcal{N}$ such that there exists a smooth nearest point projection map $\Pi_{\mathcal{N}}$ from $\mathcal{N}_{\delta}$, the $\delta$ neighborhood of $\mathcal{N}$, to $\mathcal{N}$, see also Sim96, Appendix 2.12.3]. Note that $P_{\mathcal{N}}(y)=$ $\nabla \Pi_{\mathcal{N}}(y)$ for $y \in \mathcal{N}$. We revisit this fact in Lemma 5.1
} 
Note that from the definitions of $u^{h}$ and $v^{h}$, and (7) that there exists $C>0$ such that for any $0<T<+\infty$

$$
\int_{0}^{T} \int_{\Omega}\left|u^{h}-v^{h}\right|^{2} \leq C h \sum_{i=1}^{\left[\frac{T}{h}\right]+1} \int_{\Omega}\left|u^{i+1}-u^{i}\right|^{2} \leq C h^{2} E_{s, p}\left(u_{0}\right),
$$

where $\left[\frac{T}{h}\right]$ denotes the largest integer part of $\frac{T}{h}$. As an immediate consequence of (11), we obtain that $u \equiv v$ in $\Omega \times(0, \infty)$. Moreover, since $u^{h}(x, t) \in \mathcal{N}$ for a.e. $(x, t) \in \Omega \times(0, \infty)$, (11) also implies that $u(x, t) \in \mathcal{N}$ for a.e. $(x, t) \in \Omega \times(0, \infty)$. By the sequential lower semicontinuity of $E_{s, p}$ and (8), we conclude that for a.e. $t>0$, it holds

$$
E_{s, p}(u(\cdot, t))+\frac{1}{2} \int_{0}^{t} \int_{\Omega}\left|\partial_{t} u\right|^{2} \leq E_{s, p}\left(u_{0}\right) .
$$

In particular, $u \in L^{\infty}\left((0, \infty), W^{s, p}(\Omega, \mathcal{N})\right)$ and $\partial_{t} u \in L^{2}(\Omega \times(0, \infty))$.

Finally, it follows from the Euler-Lagrange equation (6) that

$$
\begin{gathered}
\int_{\Omega_{T}^{2}} \frac{\left|u^{h}(x, t)-u^{h}(y, t)\right|^{p-2}\left(u^{h}(x, t)-u^{h}(y, t)\right)(\varphi(x, t)-\varphi(y, t))}{|x-y|^{n+s p}} \\
+\int_{\Omega_{T}} \partial_{t} v^{h}(x, t) \varphi(x, t)=0,
\end{gathered}
$$

for any $\varphi \in L^{\infty}\left(0, T ; W_{0}^{s, p}\left(\Omega, T_{u^{h}(\cdot, t)} \mathcal{N}\right)\right) \cap L^{\infty}\left(\Omega_{T}, \mathbb{R}^{L}\right)$. 3

Step 2: $u$ satisfies the equation (5) in the sense of distributions when $\mathcal{N}=\mathbb{S}^{L-1}$ or when $\mathcal{N}$ is a compact homogenous Riemannian manifold. In the case of the sphere, this follows from the arguments in Section 4 . For homogeneous Riemannian manifolds see Section 5 .

\section{Proof of Theorem 1.1: Step 2 - the CASE of A SPhere}

Let $u$ be constructed as in Section 3. Assume that $\mathcal{N}=\mathbb{S}^{L-1}$. Then $u$ satisfies the equation (5) in the sense of distributions when $\mathcal{N}=\mathbb{S}^{L-1}$. That is, for any $0<T<\infty$,

$$
\int_{\Omega_{T}}\left\langle\partial_{t} u(x, t), \varphi(x, t)\right\rangle+\int_{0}^{T} E_{s, p}^{\prime}(u(\cdot, t), \varphi(\cdot, t))=0,
$$

for all $\varphi \in L^{\infty}\left(0, T ; W_{0}^{s, p}\left(\Omega, T_{u(\cdot, t)} \mathbb{S}^{L-1}\right)\right) \cap L^{\infty}\left(\Omega_{T}, \mathbb{R}^{L}\right)$.

For any $\psi \in L^{\infty}\left(0, T ; W_{0}^{s, p}\left(\Omega, \mathbb{R}^{L}\right)\right) \cap L^{\infty}\left(\Omega_{T}, \mathbb{R}^{L}\right)$, we can check that

$$
\varphi^{h}=u^{h} \times \psi \in L^{\infty}\left(0, T ; W_{0}^{s, p}\left(\Omega, T_{u^{h}(\cdot, t)} \mathbb{S}^{L-1}\right)\right) \cap L^{\infty}\left(\Omega_{T}, \mathbb{R}^{L}\right) .
$$

\footnotetext{
${ }^{3}$ Here we denote the Sobolev space $W_{0}^{s, p}\left(\Omega, T_{u^{h}(\cdot, t)} \mathbb{S}^{L-1}\right):=\left\{\psi \in W_{0}^{s, p}\left(\Omega, \mathbb{R}^{L}\right): \psi(x, t) \in T_{u^{h}(x, t)} \mathbb{S}^{L-1}\right.$ a.e. $\left.x \in \Omega\right\}$.
} 
Substituting $\varphi^{h}$ into (13), we obtain that

$$
\int_{\Omega_{T}}\left\langle\partial_{t} v^{h}(x, t), u^{h}(\cdot, t) \times \psi(\cdot, t)\right\rangle+\int_{0}^{T} E_{s, p}^{\prime}\left(u^{h}(\cdot, t), u^{h}(\cdot, t) \times \psi(\cdot, t)\right)=0 .
$$

It is readily seen that

$$
\int_{\Omega_{T}}\left\langle\partial_{t} v^{h}(x, t), u^{h}(\cdot, t) \times \psi(\cdot, t)\right\rangle \stackrel{h \rightarrow 0}{\longrightarrow} \int_{\Omega_{T}}\left\langle\partial_{t} u(x, t), u(\cdot, t) \times \psi(\cdot, t)\right\rangle .
$$

Now we want to apply Lemma 2.2 and the symmetry of $\mathbb{S}^{L-1}$ to show that

$$
\int_{0}^{T} E_{s, p}^{\prime}\left(u^{h}(\cdot, t), u^{h}(\cdot, t) \times \psi(\cdot, t)\right) \stackrel{h \rightarrow 0}{\longrightarrow} \int_{0}^{T} E_{s, p}^{\prime}(u(\cdot, t), u \times \psi(\cdot, t)) .
$$

To show (15), first note that

$$
\begin{aligned}
\left\langle u^{h}(x, t)-u^{h}(y, t), u^{h}(x, t) \times \psi(x, t)-u^{h}(y, t) \times \psi(y, t)\right\rangle \\
=\left\langle\left(u^{h}(x, t)-u^{h}(y, t)\right) \times u^{h}(x, t), \psi(x, t)\right\rangle \\
\quad-\left\langle\left(u^{h}(x, t)-u^{h}(y, t)\right) \times u^{h}(y, t), \psi(y, t)\right\rangle \\
=\left\langle\left(u^{h}(x, t)-u^{h}(y, t)\right) \times u^{h}(x, t), \psi(x, t)\right\rangle \\
\quad-\left\langle\left(u^{h}(x, t)-u^{h}(y, t)\right) \times u^{h}(x, t), \psi(y, t)\right\rangle \\
=\left\langle\left(u^{h}(x, t)-u^{h}(y, t)\right) \times u^{h}(x, t), \psi(x, t)-\psi(y, t)\right\rangle,
\end{aligned}
$$

where we have used the fact

$$
\left(u^{h}(x, t)-u^{h}(y, t)\right) \times\left(u^{h}(x, t)-u^{h}(y, t)\right)=0 .
$$

For $v: \Omega_{T} \rightarrow \mathbb{R}^{L}$, set

$$
\mathcal{C}^{p}[v](x, y, t)=\frac{|v(x, t)-v(y, t)|^{p-2}}{|x-y|^{n+s p}}, \quad(x, y, t) \in \Omega_{T}^{2} .
$$

Then we can rewrite $\int_{0}^{T} E_{s, p}^{\prime}\left(u^{h}(\cdot, t), u^{h}(\cdot, t) \times \psi(\cdot, t)\right)$ as follows.

$$
\begin{aligned}
& \int_{0}^{T} E_{s, p}^{\prime}\left(u^{h}(\cdot, t), u^{h}(\cdot, t) \times \psi(\cdot, t)\right)= \\
& \int_{\Omega_{T}^{2}} \mathcal{C}^{p}\left[u^{h}\right](x, y, t)\left\langle\left(u^{h}(x, t)-u^{h}(y, t)\right) \times u^{h}(x, t), \psi(x, t)-\psi(y, t)\right\rangle .
\end{aligned}
$$

\footnotetext{
${ }^{4}$ See Che89 for the heat flow of harmonic maps and Sha98 for wave maps to $\mathbb{S}^{L-1}$.
} 
From Lemma 2.2 and (16), we see that

$$
\begin{aligned}
& \int_{\Omega_{T}^{2}} \mathcal{C}^{p}\left[u^{h}\right](x, y, t)\left\langle\left(u^{h}(x, t)-u^{h}(y, t)\right) \times u^{h}(x, t), \psi(x, t)-\psi(y, t)\right\rangle \\
& \stackrel{h \rightarrow 0}{\longrightarrow} \int_{\Omega_{T}^{2}} \mathcal{C}^{p}[u](x, y, t)\langle(u(x, t)-u(y, t)) \times u(x, t), \psi(x, t)-\psi(y, t)\rangle \\
& \quad=\int_{0}^{T} E_{s, p}^{\prime}(u(\cdot, t), u(\cdot, t) \times \psi(\cdot, t)) .
\end{aligned}
$$

Thus we obtain that

$$
\begin{aligned}
& \int_{\Omega_{T}}\left\langle\partial_{t} u(x, t), u(\cdot, t) \times \psi(\cdot, t)\right\rangle+\int_{0}^{T} E_{s, p}^{\prime}(u(\cdot, t), u(\cdot, t) \times \psi(\cdot, t)) \\
& =0
\end{aligned}
$$

holds for all $\psi \in L^{\infty}\left(0, T ; W_{0}^{s, p}\left(\Omega, \mathbb{R}^{L}\right)\right) \cap L^{\infty}\left(\Omega_{T}, \mathbb{R}^{L}\right)$.

Finally, we claim that (17) implies (14). In fact, for any $\varphi \in$ $L^{\infty}\left(0, T ; W_{0}^{s, p}\left(\Omega, T_{u(\cdot, t)} \mathbb{S}^{L-1}\right)\right) \cap L^{\infty}\left(\Omega_{T}, \mathbb{R}^{L}\right)$, if we define

$$
\psi(\cdot, t)=-u(\cdot, t) \times \varphi(\cdot, t),
$$

then we have $\psi \in L^{\infty}\left(0, T ; W_{0}^{s, p}\left(\Omega, \mathbb{R}^{L}\right)\right) \cap L^{\infty}\left(\Omega_{T}, \mathbb{R}^{L}\right)$, and

$$
\begin{aligned}
& u(\cdot, t) \times \psi(\cdot, t)=(u(\cdot, t) \times \varphi(\cdot, t)) \times u(\cdot, t) \\
& =\langle u(\cdot, t), u(\cdot, t)\rangle \varphi(\cdot, t)-\langle u(\cdot, t), \varphi(\cdot, t)\rangle u(\cdot, t) \\
& =\varphi(\cdot, t),
\end{aligned}
$$

where we have used $\langle u(\cdot, t), u(\cdot, t)\rangle=0$, and $\langle u(\cdot, t), \varphi(\cdot, t)\rangle=0$ since $\varphi(\cdot, t) \in T_{u(\cdot, t)} \mathbb{S}^{L-1}$. Thus (14) follows from (17). This completes the proof when $\mathcal{N}=\mathbb{S}^{L-1}$. We will give a proof for $\mathcal{N}$ a compact Riemannian homogeneous manifold in the next section.

\section{Proof of Theorem 1.1: Step 2 - the CASE of HOMOGENEOUS RIEMANNIAN MANIFOLDS}

In this section, we will show that theorem 1.1 also holds when the target manifold $\mathcal{N}$ is a compact Riemannian homogeneous manifold that is equivariantly embedded in $\mathbb{R}^{L}$.

First we recall the following property on compact Riemannian homogeneous manifolds, which was proven by [MS80, see also [Fre96].

Theorem 5.1 (Moore-Schlafly). For any compact Riemannian manifold $\mathcal{N}$ with a compact Lie group $G$ acting on it by isometries we find for some $L \in \mathbb{N}$ an orthogonal representation $\rho$ of $G$ to the isometric group of an Euclidean space $\mathbb{R}^{L}$ and an isometric embedding $\Psi: \mathcal{N} \rightarrow \mathbb{R}^{L}$ 
which is equivariant with respect to $\rho$, that is $\Psi(g p)=\rho(g) \Psi(p)$ for all $p \in \mathcal{N}$ and $g \in G$.

With Theorem 5.1, we can follow the arguments by Fre96 pages 527-528 to show that, by assuming $\mathcal{N} \subset \mathbb{R}^{L}$ and $G \subset \operatorname{Iso}\left(\mathbb{R}^{L}\right)$, the isometric group of $\mathbb{R}^{L}$, for any $G$-killing vector field $X$ on $\mathcal{N}$, there exists a killing vector field $\widehat{X}$ on $\mathbb{R}^{L}$ with respect to $\operatorname{Iso}\left(\mathbb{R}^{L}\right)$ such that

$$
X=\left.\widehat{X}\right|_{\mathcal{N}}
$$

It follows from [Hél91] Lemma 2 that there exist a family of $G$-killing vector fields $\left\{X_{\alpha}\right\}_{\alpha=1}^{l}$ on $\mathcal{N}$, with $l=\operatorname{dim}(G)$, and another family of smooth vector fields $\left\{Y_{\alpha}\right\}_{\alpha=1}^{l}$ on $\mathcal{N}$ such that for any $y \in \mathcal{N}$, it holds that

$$
v=\sum_{\alpha=1}^{l}\left\langle X_{\alpha}(y), v\right\rangle Y_{\alpha}(y) \quad \forall v \in T_{y} \mathcal{N} .
$$

We use crucially the following property of our Killing fields:

$$
\left\langle X_{\alpha}(p)-X_{\alpha}(q), p-q\right\rangle=0 \quad \forall p, q \in \mathcal{N}, \alpha=1, \ldots, l .
$$

Proof of (19). In fact, let $\left\{\widehat{X}_{\alpha}\right\}_{\alpha=1}^{p}$ be a family of killing vector fields on $\mathbb{R}^{L}$, with respect to Iso $\left(\mathbb{R}^{L}\right)$, such that

$$
X_{\alpha}(y)=\widehat{X_{\alpha}}(y), \forall y \in \mathcal{N}, 1 \leq \alpha \leq l \text {. }
$$

Then we have

$$
\begin{aligned}
\left\langle X_{\alpha}(p)-X_{\alpha}(q), p-q\right\rangle & =\left\langle\widehat{X_{\alpha}}(p)-\widehat{X_{\alpha}}(q), p-q\right\rangle \\
& =\left\langle D \widehat{X_{\alpha}}\left(p_{*}\right)(p-q), p-q\right\rangle \\
& =0,
\end{aligned}
$$

for some point $p_{*}$ in the line segment $[p, q]$, where we have used the fact that $D \widehat{X_{\alpha}}$ is skew-symmetric in the last step.

We also need the following welll-known fact. See also Sim96, Appendix 2.12.3].

Lemma 5.1. Let $\Pi_{\mathcal{N}}: \mathcal{N}_{\delta} \rightarrow \mathcal{N} \subset \mathbb{R}^{L}$ be the orthogonal projection from the $\delta$-tubular neighbourhood of $\mathcal{N}$ into $\mathcal{N}$ for some small $\delta>0$. For $p \in \mathcal{N}$, set $P_{\mathcal{N}}(p) \in \mathbb{R}^{L \times L}$ by

Then

$$
\left.P_{\mathcal{N}}(p)(v) \equiv \frac{d}{d t}\right|_{t=0} \Pi_{\mathcal{N}}(p+t v), v \in \mathbb{R}^{L} .
$$

$$
P_{\mathcal{N}}(p)(v)=v \quad \forall v \in T_{p} \mathcal{N}, \quad P_{\mathcal{N}}(p)(v)=0 \quad \forall v \in\left(T_{p} \mathcal{N}\right)^{\perp} .
$$

In particular, $P_{\mathcal{N}}(p)^{2}=P_{\mathcal{N}}(p)$ and $\left(P_{\mathcal{N}}(p)\right)^{T}=P_{\mathcal{N}}(p)$ for any $p \in \mathcal{N}$. 
Proof. Indeed, fix $p \in \mathcal{N}$, and let us write $P$ instead of $P_{\mathcal{N}}(p)$. Let $o_{1}, \ldots, o_{l}$ be an orthonormal basis of $T_{p} \mathcal{N}$ and $o_{l+1}, \ldots, o_{L}$ be an orthonormal basis of $\left(T_{p} \mathcal{N}\right)^{\perp}$. Then, since $P o_{\beta}=P o_{\alpha}=0$ for $\alpha, \beta \in$ $\{l+1 \ldots N\}$,

$$
\left\langle P o_{\alpha}, o_{\beta}\right\rangle=0=\left\langle o_{\alpha}, P o_{\beta}\right\rangle \quad \forall \alpha, \beta=l+1, \ldots, N .
$$

Also, since $P o_{\alpha}=o_{\alpha}$ for $\alpha=1, \ldots, l$,

$$
\left\langle P o_{\alpha}, o_{\beta}\right\rangle=\left\langle o_{\alpha}, o_{\beta}\right\rangle=0=\left\langle o_{\alpha}, P o_{\beta}\right\rangle \quad \forall \alpha=1, \ldots l ; \beta=l+1, \ldots, N .
$$

Finally,

$$
\left\langle P o_{\alpha}, o_{\beta}\right\rangle=\left\langle o_{\alpha}, o_{\beta}\right\rangle=\left\langle o_{\alpha}, P o_{\beta}\right\rangle \quad \forall \alpha, \beta=1, \ldots l .
$$

Finally let $v, w \in \mathbb{R}^{n}$ then we have $v=\sum_{\alpha=1}^{N} \lambda_{\alpha} o_{\alpha}, w=\sum_{\beta=1}^{N} \mu_{\beta} o_{\beta}$. Consequently,

$$
\langle P v, w\rangle=\sum_{\alpha, \beta=1}^{N} \lambda_{\alpha} \mu_{\beta}\left\langle P o_{i}, o_{j}\right\rangle=\sum_{\alpha, \beta=1}^{N} \lambda_{\alpha} \mu_{\beta}\left\langle o_{\alpha}, P o_{\beta}\right\rangle=\langle P v, w\rangle .
$$

Thus, $P$ is symmetric.

Note that then for any $v, w \in \mathbb{R}^{N}$, since $P_{\mathcal{N}}^{T}=P_{\mathcal{N}}$ and $P_{\mathcal{N}} X_{\alpha}=X_{\alpha}$, it follows from (18) that

$$
\begin{aligned}
w^{T} P_{\mathcal{N}} v & =\sum_{\alpha=1}^{l}\left\langle X_{\alpha}, P_{\mathcal{N}} v\right\rangle\left\langle Y_{\alpha}, w\right\rangle=\sum_{\alpha=1}^{l}\left\langle P_{\mathcal{N}} X_{\alpha}, v\right\rangle\left\langle Y_{\alpha}, w\right\rangle \\
& =\sum_{\alpha=1}^{l}\left\langle X_{\alpha}, v\right\rangle\left\langle Y_{\alpha}, w\right\rangle .
\end{aligned}
$$

Thus,

$$
\left(P_{\mathcal{N}}(p)\right)_{i j}=\sum_{\alpha=1}^{l} X_{\alpha}^{i}(p) Y_{\alpha}^{j}(p)=\sum_{\alpha=1}^{l} Y_{\alpha}^{i}(p) X_{\alpha}^{j}(p), \forall p \in \mathcal{N},
$$

where the last equality holds because $P(p)_{i j}=P(p)_{j i}$.

With these preparations, we are ready to show Step 2 in the proof of Theorem 1.1 for a Riemannian homogeneous manifold $\mathcal{N}$ that is equivariantly embedded into $\mathbb{R}^{L}$.

Let $v^{h}, u^{h}, u$ be as constructed in Step 1, Section 3 .

It suffices to show that for any $0<T<\infty$,

$$
\int_{\Omega_{T}}\left\langle\partial_{t} u, P_{\mathcal{N}}(u)(\varphi)\right\rangle+\int_{0}^{T} E_{s, p}^{\prime}\left(u(\cdot, t), P_{\mathcal{N}}(u(\cdot, t))(\varphi(\cdot, t))\right)=0,
$$

for all $\varphi \in L^{\infty}\left(0, T ; W_{0}^{s, p}\left(\Omega, \mathbb{R}^{L}\right)\right) \cap L^{\infty}\left(\Omega_{T}, \mathbb{R}^{L}\right)$. 
To simplify the presentation, we set

$$
\mathcal{W}_{h}(x, y, t)=\frac{\left|u^{h}(x, t)-u^{h}(y, t)\right|^{p-2}\left(u^{h}(x, t)-u^{h}(y, t)\right)}{|x-y|^{n+s p}},
$$

and

$$
\mathcal{W}(x, y, t)=\frac{|u(x, t)-u(y, t)|^{p-2}(u(x, t)-u(y, t))}{|x-y|^{n+s p}} .
$$

From (13), we see that

$$
\begin{aligned}
& -\int_{\Omega_{T}}\left\langle\partial_{t} v^{h}(x, t), X_{\alpha}\left(u^{h}(x, t)\right) \eta(x, t)\right\rangle \\
& =\int_{\Omega_{T}^{2}}\left\langle\mathcal{W}_{h}(x, y, t), X_{\alpha}\left(u^{h}(x, t)\right) \eta(x, t)-X_{\alpha}\left(u^{h}(y, t)\right) \eta(y, t)\right\rangle
\end{aligned}
$$

holds for any $1 \leq \alpha \leq l$ and $\eta \in L^{\infty}\left(0, T ; W_{0}^{s, p}(\Omega, \mathbb{R})\right) \cap L^{\infty}\left(\Omega_{T}, \mathbb{R}\right)$.

From (19), we have

$$
\left\langle\mathcal{W}_{h}(x, y, t), X_{\alpha}\left(u^{h}(x, t)\right) \eta(x, t)\right\rangle=\left\langle\mathcal{W}_{h}(x, y, t), X_{\alpha}\left(u^{h}(y, t)\right) \eta(x, t)\right\rangle
$$

so that

$$
\begin{aligned}
& \int_{\Omega^{2}}\left\langle\mathcal{W}_{h}(x, y, t), X_{\alpha}\left(u^{h}(x, t)\right) \eta(x, t)-X_{\alpha}\left(u^{h}(y, t)\right) \eta(y, t)\right\rangle \\
& =\int_{\Omega^{2}}\left\langle\mathcal{W}_{h}(x, y, t), X_{\alpha}\left(u^{h}(y, t)\right)(\eta(x, t)-\eta(y, t))\right\rangle \\
& \stackrel{h \rightarrow 0}{\longrightarrow} \int_{\Omega^{2}}\left\langle\mathcal{W}(x, y, t), X_{\alpha}(u(y, t))(\eta(x, t)-\eta(y, t))\right\rangle \\
& =\int_{\Omega^{2}}\left\langle\mathcal{W}(x, y, t), X_{\alpha}(u(x, t)) \eta(x, t)-X_{\alpha}(u(y, t)) \eta(y, t)\right\rangle,
\end{aligned}
$$

where we have used Lemma 2.2 and (19) in the last two steps.

It is straightforward that

$$
\begin{aligned}
& \int_{\Omega_{T}}\left\langle\partial_{t} v^{h}(x, t), X_{\alpha}\left(u^{h}(x, t)\right) \eta(x, t)\right\rangle \\
& \stackrel{h \rightarrow 0}{\longrightarrow} \int_{\Omega_{T}}\left\langle\partial_{t} u(x, t), X_{\alpha}(u(x, t)) \eta(x, t)\right\rangle .
\end{aligned}
$$

Equalling these two limits yields that

$$
\begin{aligned}
& \int_{\Omega_{T}}\left\langle\partial_{t} u(x, t), X_{\alpha}(u(x, t)) \eta(x, t)\right\rangle+ \\
& \int_{\Omega_{T}^{2}}\left\langle\mathcal{W}(x, y, t), X_{\alpha}(u(x, t)) \eta(x, t)-X_{\alpha}(u(y, t)) \eta(y, t)\right\rangle=0
\end{aligned}
$$

for all $1 \leq \alpha \leq l$ and $\eta \in L^{\infty}\left(0, T ; W_{0}^{s, p}(\Omega, \mathbb{R})\right) \cap L^{\infty}\left(\Omega_{T}, \mathbb{R}\right)$. 
Now for any $\varphi \in L^{\infty}\left(0, T ; W_{0}^{s, p}\left(\Omega, \mathbb{R}^{L}\right)\right) \cap L^{\infty}\left(\Omega_{T}, \mathbb{R}^{L}\right)$ and $1 \leq \alpha \leq l$ substitute $\eta=\eta_{\alpha} \equiv\left\langle Y_{\alpha}(u), \varphi\right\rangle$ into (22) and take summation of the resulting equations over $1 \leq \alpha \leq l$. Observe that in view of (20),

$$
\sum_{\alpha=1}^{l} X_{\alpha}(u(x, t)) \eta_{\alpha}(x, t)=P(u(x, t)) \varphi(x, t) .
$$

Thus, we arrive at

$$
\begin{aligned}
& \int_{\Omega_{T}}\left\langle\partial_{t} u(x, t), P(u(x, t)) \varphi(x, t)\right\rangle \\
= & \int_{\Omega_{T}^{2}}\langle\mathcal{W}(x, y, t), P(u(x, t)) \varphi(x, t)-P(u(y, t)) \varphi(y, t)\rangle=0,
\end{aligned}
$$

for any $\varphi \in L^{\infty}\left(0, T ; W_{0}^{s, p}\left(\Omega, \mathbb{R}^{L}\right)\right) \cap L^{\infty}\left(\Omega_{T}, \mathbb{R}^{L}\right)$. This shows (21) and thus completes the proof of Theorem 1.1 for a Riemannian homogeneous manifold $\mathcal{N}$.

\section{REFERENCES}

[Che89] Yun Mei Chen. The weak solutions to the evolution problems of harmonic maps. Math. Z., 201(1):69-74, 1989.

[CHH94] Yun Mei Chen, Min Chun Hong, and Norbert Hungerbühler. Heat flow of $p$-harmonic maps with values into spheres. Math. Z., 215(1):25-35, 1994.

[CS89] Yun Mei Chen and Michael Struwe. Existence and partial regularity results for the heat flow for harmonic maps. Math. Z., 201(1):83-103, 1989.

[DL13] F. Da Lio. Fractional harmonic maps into manifolds in odd dimension $n>1$. Calc. Var. PDE, 48(3-4):421-445, 2013.

[DLR11a] Francesca Da Lio and Tristan Rivière. Sub-criticality of non-local Schrödinger systems with antisymmetric potentials and applications to half-harmonic maps. Adv. Math., 227(3):1300-1348, 2011.

[DLR11b] Francesca Da Lio and Tristan Rivière. Three-term commutator estimates and the regularity of $\frac{1}{2}$-harmonic maps into spheres. Anal. PDE, 4(1):149-190, 2011.

[Fre96] Alex Freire. Global weak solutions of the wave map system to compact homogeneous spaces. Manu. Math., 91(4):525-533, 1996.

[Hél91] F. Hélein. Regularity of weakly harmonic maps from a surface into a manifold with symmetries. Manuscripta Math, 70(2):203-218, 1991.

[HL89] Robert Hardt and Fang-Hua Lin. Partially constrained boundary conditions with energy minimizing mappings. Comm. Pure Appl. Math., 42(3):309-334, 1989.

[LW08] Fanghua Lin and Changyou Wang. The analysis of harmonic maps and their heat flows. World Scientific Publishing Co. Pte. Ltd., Hackensack, NJ, 2008. 
[MS80] J.D. Moore and R. Schlafly. On equivariant isometric embeddings. Math. Z., 173:119-133, 1980.

[MS15] Vincent Millot and Yannick Sire. On a fractional Ginzburg-Landau equation and 1/2-harmonic maps into spheres. Arch. Ration. Mech. Anal., $215(1): 125-210,2015$.

[PG11] X. Pu and B. Guo. The fractional Landau-Lifshitz-Gilbert equation and the heat flow of harmonic maps. Calc. Var. Partial Differential Equations, 42(1-2):1-19, 2011.

[Sch12] A. Schikorra. Regularity of n/2-harmonic maps into spheres. J. Differential Equations, 252:1862-1911, 2012.

[Sch15] A. Schikorra. Integro-differential harmonic maps into spheres. Comm.PDE, 40(1):506-539, 2015.

[Sha98] J. Shatah. Weak solutions and development of singularities of the $s u(2)-$ $\sigma$ model. Comm. Pure Appl. Math., 41:459-469, 1998.

[Sim96] L. Simon. Theorems on regularity and singularity of energy minimizing maps. Lectures in Mathematics ETH Zürich. Birkhäuser Verlag, Basel, 1996. Based on lecture notes by Norbert Hungerbühler.

[Str88] Michael Struwe. On the evolution of harmonic maps in higher dimensions. J. Differential Geom., 28(3):485-502, 1988.

UNIVERSITY OF BASEL

E-mail address: armin.schikorra@unibas.ch

Johns Hopkins University, Krieger Hall, Baltimore, USA

E-mail address: sire@math.jhu.edu

Purdue University, USA

E-mail address: wang2482@purdue.edu 